\title{
Diagnosis of Alzheimer's disease with [18F]PET in mild and asymptomatic stages
}

\author{
Alexander Drzezga ${ }^{\mathrm{a}, \mathrm{b}, *}$ \\ ${ }^{a}$ Harvard Medical School, Massachusetts General Hospital, Athinoula A. Martinos Center for Biomedical \\ Imaging, Charlestown, MA, USA \\ ${ }^{\mathrm{b}}$ Department of Nuclear Medicine, Klinikum rechts der Isar, Technische Universität München, Munich, Germany
}

\begin{abstract}
With longer life expectancy, dementia based on the age-related Alzheimers' disease (AD) has turned into one of the most prevalent disorders of older age, representing a serious medical and socio-economic issue. There has been growing interest in early diagnosis of this disease, particularly regarding the initiation of new treatment strategies ahead of the onset of irreversible neuronal damage. It is accepted that the pathologic changes underlying $\mathrm{AD}$ appear in the brain years to decades before the symptomatic stages. Consequently, clinical measures of cognitive impairment, as used for definition of dementia, will not allow early diagnosis of AD-pathology in the mild or asymptomatic stages. Thus, a need for complementary sensitive biomarkers is apparent. Brain imaging markers are among the most promising candidates for this diagnostic challenge. Particularly, [18F]FDG PET as a marker of regional neuronal function has been demonstrated to represent a most sensitive and specific method for early identification of AD-pathology and thus for prediction of dementia of the Alzheimer type (DAT), even in the mild and asymptomatic stages. Currently, systematic data of comparable quality are hardly available for any other imaging procedure. The purpose of this article is to describe the typical findings of [18F]FDG PET in different stages of AD and to demonstrate its value for early and reliable diagnosis of Alzheimer's disease, particularly ahead of the stage of dementia of the Alzheimer's type.
\end{abstract}

\section{Introduction}

Age represents the greatest known risk factor for dementia. The prevalence of the disease shows an almost exponential increase with age [10]. More than $20 \%$ of persons older than 80 years are affected by manifest dementia $[9,36]$ and the risk doubles approximately every 5 years [2,119]. Most of these dementia cases of older age are based on the neurodegenerative disorder Alzheimer's disease (AD) [14]. With increasing lifespan, dementia turns into one of the most prevalent and debilitating disorders in today's societies, characterized by an insidious onset and a progressive impairment of cognitive functions, finally leading to an impairment in the capabilities of daily living $[33,60]$. Only in the

*Address for correspondence: Priv. Doz. Dr. Alexander Drzezga, MD, Nuklearmedizinische Klinik, Klinikum rechts der Isar der Technischen Universität München, Ismaninger Str. 22, 81675 München, Germany. Tel.: +49 894140 2971; Fax: +49 894140 4841; E-mail: a.drzezga@1rz.tu-muenchen.de.
United States up to 5 million individuals are estimated to suffer from Alzheimer's disease today [1]. AD already represents the fifth leading cause of death in Americans older than the age of 65 years. Regarding the fact that the elderly population (those aged 65 years or older) in the USA is expected to duplicate from approximately 35 million today to more than 70 million by 2030 , the incidence of Alzheimer's disease is expected to approach nearly a million people per year, with a total estimated prevalence of 11 to 16 million persons by 2050 [87] These facts illustrate that this disorder will represent an enormous burden in the future, not only for the patients and their relatives but also regarding socio-economic and health care systems.

Today's diagnostic algorithms for the diagnostic verification of $\mathrm{AD}$ are still mainly directed towards the definition of manifest dementia based on clinical symptoms [60]. The diagnostic standards of the NINCDSADRDA rely on the exclusion of other causes for dementia, leaving AD as the end point of a long diagnostic road with neuropsychological examination as the 
most important tool. These tools unfortunately have a rather low sensitivity and specificity with $80 \%$ and $70 \%$ respectively- these numbers might even be lower particularly when looking at the crucial early stages of AD [52]. The systematic algorithm of the clinical diagnosis of dementia is currently divided into two steps: 1.) Confirmation of the diagnosis "dementia" and 2.) Differential diagnosis of the cause underlying the dementia. Step one is usually carried out on the basis of an interview with the patient and usually also with at least one informant (e.g. spouse or children of the patient), as well as by clinical neuropsychological testing of the patient. The second step also includes laboratory tests and other technical examinations such as ECG and brain imaging procedures. According to current official guidelines, the performance of a cerebral CT or MRI-scan is regarded mandatory at this step [52]. The purpose of these morphologic imaging procedures is mainly to exclude non-neurodegenerative causes of cognitive impairment, such as brain tumors or vascular abnormalities. Following the exclusion of other potential causes, the diagnostic assignment to dementia of the Alzheimer type (DAT) or to another neurodegenerative disorder will usually mostly result on the basis of the type of cognitive symptoms.

A definite diagnosis of Alzheimer's disease (AD), however, is currently only possible by post mortem histopathological analysis of the brain. The hallmarks of histological verification of $\mathrm{AD}$ are the deposits of $ß$-amyloid plaques and neurofibrillary tangles [12]. In contrast to other disease entities, the in vivo extraction of tissue for histopathology (e.g. by brain biopsy) can hardly be reasonably justified for diagnosis of dementia. Besides this limitation regarding the definite diagnosis of $\mathrm{AD}$ by neuropsychological tools is limited. It is well accepted that the pathology of AD starts years to decades before the onset of cognitive symptoms. Out of this perspective clinical definition of manifest symptomatic dementia will consistently represent an already advanced stage of $\mathrm{AD}[12,19]$. In summary, the early and definite diagnosis of $\mathrm{AD}$ is hampered by the low sensitivity and specificity of clinical/neuropsychological evaluation and the limited accessibility of brain tissue for histopathological analysis. On the other hand, considerable progress has recently been made regarding insights into pathomechanisms underlying AD. This progress has been paralleled by increasing efforts to develop new treatment options, mostly directed against amyloid deposition, such as e.g. secretase blockers and immunization strategies [44]. For all causal therapeutic approaches it is of apparent importance to improve early diagnosis of the disease, ahead of the clinical stage of dementia, to allow treatment initiation in low or even asymptomatic stages, i.e. prior to irreversible neuronal damage. Furthermore, the expensive and in part also risky new therapeutic approaches will obviously demand a highly specific diagnosis and tools for monitoring treatment success. As a consequence, the need for a more reliable test for early and specific diagnosis of AD is evident. The most reasonable approach to evaluate the sensitivity of a new diagnostic tool for early diagnosis of $\mathrm{AD}$, is apparently to test its value in AD risk populations. In general, such risk populations can be selected on the basis of different approaches: a) selection of patients with mild cognitive symptoms, not yet sufficient for diagnosis of dementia or b) selection of patients without cognitive symptoms but with a genetic or familiar risk for AD. Regarding a), a diagnostic entity has been created established in the recent years, termed MCI for "mild cognitive impairment". This clinically defined group encloses patients with cognitive symptoms, which do not yet affect their activities of daily living. It has been shown that 15-30\% of these patients will develop manifest DAT within a short period of time. Thus, MCI is regarded to define represent a risk population for $\mathrm{AD}$ [81]. However, not all MCI-patients will develop DAT and today a definite diagnosis of $\mathrm{AD}$ in the stage of MCI is not reliably possible based on neuropsychological tests alone. Regarding b), several known genetic risk factors exist. The Apolipoprotein E (ApoE) e4-allele represents the greatest known genetic risk factor for sporadic AD [30]. However, these genetic risk factors do only indicate a theoretical risk and there is no proof for the actual presence of a neurodegenerative disorder. Many carriers of the e4-allele will never develop DAT.

Consequently, the search for specific surrogate markers allowing non-invasive early and specific diagnosis of $\mathrm{AD}$ in the stage of MCI or even in asymptomatic stages of the disease has been intensified. Modern imaging procedures hold considerable promise in this context. It has been shown that imaging technologies, such as Magnetic Resonance Imaging (MRI) and Positron Emission Tomography (PET), allow the detection of structural, functional and molecular pathological changes associated with the disease progression and may be superior to neuropsychological testing regarding early and reliable diagnosis of AD [85,118]. As mentioned above, morphological imaging procedures, such as CT and MRI are already an essential part of the standard diagnostic procedure. These techniques can exclude several non-neurodegenerative causes for 
symtomatic dementia, such as vascular and tumorous diseases or normal pressure hydrocephalus [110]. Furthermore, particularly MRI is capable of precise measurements of regional brain atrophy, e.g. of the hippocampal region. However, morphological changes do show some overlap between different neurodegenerative disorders and the specific proof of a certain neurodegenerative process on the basis of certain patterns of atrophy may be less reliable. In addition, it seems that morphological changes appear later in the progress of disease, subsequently to the onset of neuropathological and functional changes and even to the early symptomatic phase of AD [111]. Thus, morphological structural CT and MRI may not represent the optimal tools for specific differentiation of $\mathrm{AD}$ from other types of neurodegeneration so far and particularly not regarding the sensitive early diagnosis of AD in asymptomatic or MCI-stage. Advanced MRI-techniques such as repeated scanning for the longitudinal assessment of changes over time or modern spectroscopy techniques may hold additional options but still need to be evaluated systematically in larger studies.

In contrast to the mentioned morphological structural imaging procedures, functional imaging techniques such as PET allow the sensitive verification of subtle changes in brain physiology. In particular, PET imaging with the glucose analogue [18F]FDG has been demonstrated to visualize even a subtle minor decline of regional neuronal function, which may be one of the very first abnormalities accompanying the onset of AD-pathology in the brain. This imaging tool holds promise to allow a positive proof of AD in very early stages, i.e. prior to major clinical changes and changes in brain structure. However, in current guidelines, functional imaging techniques such as PET are currently still regarded as optional complementary procedures in the diagnostic setup. For several reasons this may have to be reconsidered. First, diagnosis of manifest dementia may have to be replaced by diagnosis of ongoing neurodegeneration in the future. Second, a lot of additional data, confirming the value of the existing procedures such as [18F]FDG PET has been collected. In the current article, the potential value of [18F]FDG, representing one of the most promising and well-established PET techniques, will be discussed with particular regard to early diagnosis of AD in stages ahead of clinically manifest dementia.

\section{Basics of [18F]FDG PET imaging in AD}

Current theories regarding the pathology of ADdevelopment can not be addressed here in detail. In short, AD is today most commonly regarded as a protein aggregation disorder, with extracellular aggregation of $B$-amyloid peptide and intracellular aggregation of the tau-protein being the earliest known pathological events of this disease. The $B$-amyloid peptides are cleaved from a membrane protein called APP (amyloid precursor protein) and tend to aggregate extracellularly with different affinity, depending on the length the different forms. As a consequence of aggregation, dimers, oligomeres and finally extracellular B-amyloid plaques are generated. Inside the cell, following hyperphosphorylation the tau-protein, which is usually associated with intracellular microtubuli, aggregates to the so called neurofibrillary tangles. Although the exact mechanism is not yet known, it is assumed that these aggregation pathologies lead to impaired synaptic function, neuronal dysfunction, synaptic loss and finally to neuronal death and resulting brain atrophy [36,99]. Some studies were able to confirm that neuronal loss appears later in the disease progress, when compared to tau and amyloid aggregation pathologies [88]. On the basis of this disease concept, reduced neuronal activity can apparently be regarded as a very early pathology of $\mathrm{AD}$, advancing measurable reductions of brain tissue.

Thus, measuring neuronal dysfunction does obviously represent a promising approach for early detection of $\mathrm{AD}$. With the radiolabeled glucose analogue, [18F]Fluorodeoxyglucose ([18F]FDG), a PET-tracer for in vivo assessment of neuronal function is at hand. The uptake of [18F]FDG parallels the transport of glucose into cells. Subsequently, the tracer is phosphorylized and trapped in the cell and thus allows regional assessment of regional cerebral glucose metabolism (rCGMglc) [86]. It is well known that glucose constitutes the relevant source of energy for the brain and that glucose metabolism is tightly connected to regional neuronal function. Most probably, this coupling is mediated by glial cells. Energy-demanding synaptic activity of a neuron leads to increased glucose uptake into surrounding glial cells, which subsequently transfer lactate as an energy carrier to the neuron [57]. If the $\left[{ }^{18} \mathrm{~F}\right]$ FDG PET-examination is performed in resting conditions, the tracer uptake is mainly driven by basal neuronal activity; thus it represents a measure of general neuronal integrity [95]. Inversely, impairment of neuronal function leads to a decrease of regional glucose turnover.

Consequently reductions of cerebral glucose metabolism as a measure of neuronal function in the brain in $\mathrm{AD}$ can be expected. In fact, in patients with DAT, characteristic deficits of regional glucose metabolism 


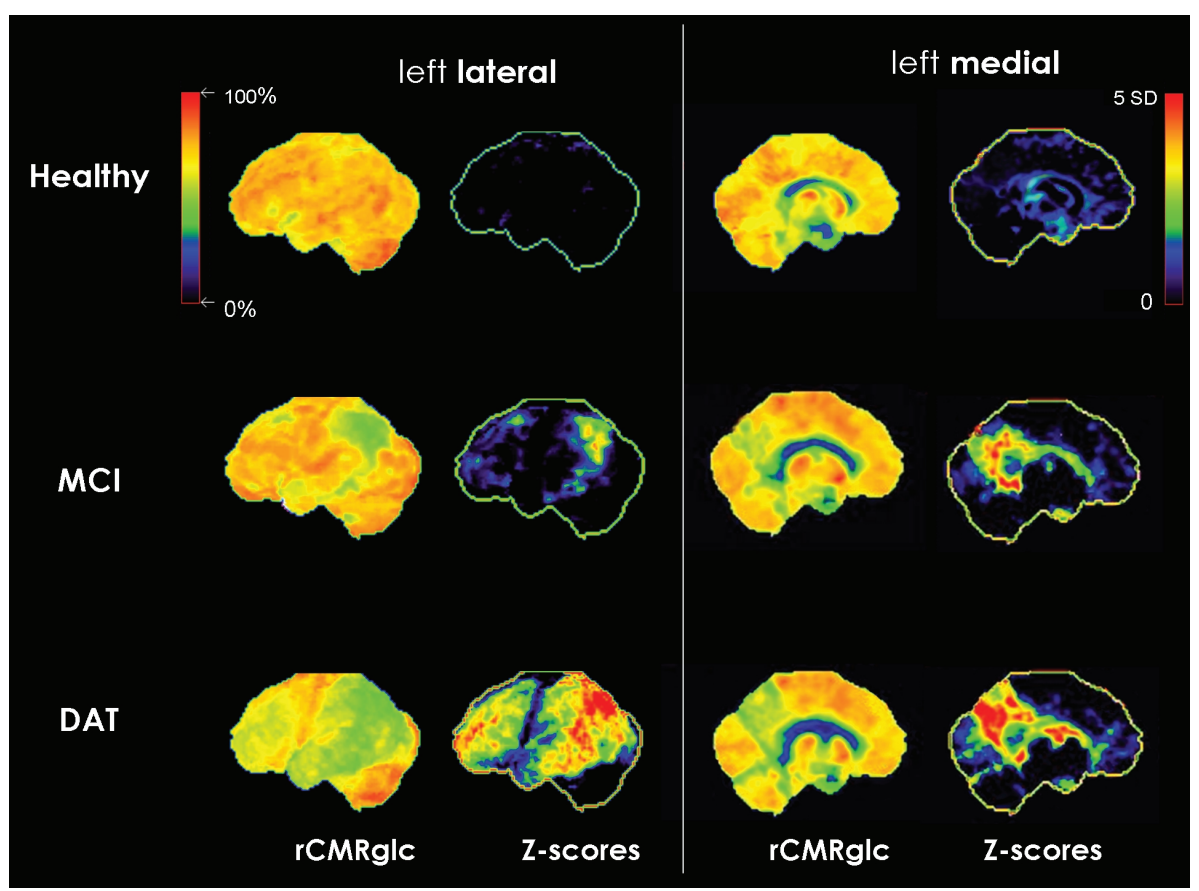

Fig. 1. Surface projections (3DSSP) of typical [18F]FDG PET findings of the left hemisphere in single subjects: healthy control (first row), MCI (second row) and DAT (third row). rCMRglc: glucose metabolism, red/yellow: normally high, green: abnormally low. Z-scores: deviations as compared with a healthy control population, yellow/red: high deviation, blue/black: low/no deviation.

in affected brain regions have often been demonstrated. Typically, temporoparietal cortex, posterior cingulate cortex and frontal cortex show hypometabolic changes. The pattern of affected brain regions fits well to the known deficits in cognitive functions, which have been associated with these particular brain regions, e.g. spatial thinking with the parietal cortex. In contrast, regions such as the sensorimotor cortex and the primary visual cortex are usually spared from hypometabolism, (see Fig. 1) corresponding to the preserved brain functions associated with these regions (vision, sensory and motor function) $[38,62,102]$. Usually patients show bilateral, but not necessarily completely symmetric hypometabolic areas. According to the disease hypothesis mentioned above, the metabolic deficits in the affected areas of the brain have been mainly assigned to reduced neuronal activity, but also to a number of further mechanisms including impaired metabolic efficiency, or $\beta$ amyloid-induced inhibition of glucose transport $[69$, 108]. Undoubtedly, disease associated loss of synapses and neuronal death would also contribute to reduced regional metabolic rates in later stages. Thus, there is still some controversy regarding the question whether reduced cerebral glucose metabolism actually represents a cause or a consequence of the disease or both. Either way, the resulting pattern of metabolic changes has a high sensitivity and specificity for AD and, thus, $\left[{ }^{18} \mathrm{~F}\right] \mathrm{FDG}$ PET does represent a valuable tool for early and also for differential diagnosis of dementia [59,62, 97,102,104].

In general, it has been demonstrated that [18F]FDGPET imaging offers a tool superior to neuropsychological testing, regarding early diagnosis of AD [85, $102,118]$. The true diagnostic quality of neuroimaging procedures can only be evaluated against histopathological assessment. In fact, several studies addressed this issue. Minoshima et al. found that patients with post-mortem histopathological proof of $\mathrm{AD}$ showed typical parietotemporal, posterior cingulate, and frontal hypometabolic changes in prior [18F]FDGPET scans [64]. Also Hoffmann et al reported that parietotemporal hypometabolism is the typical abnormality in patients with verified $\mathrm{AD}$ [42]. In a large multicenter trial Silverman et al were able to demonstrate a sensitivity of $94 \%$ and a specificity of $73 \%$ of a diagnostic setup including [18F]FDG PET regarding the diagnosis of $\mathrm{AD}$, as verified by histopathological proof of disease [102]. In contrast, in studies using histopathology as the goldstandard for neuropsychological evaluation, distinctly lower values of sensitivity $(85 \%)$ and specificity (55\%) have been found [42, 56]. These studies provide convincing evidence that 
diagnostic setups including [18F]FDG PET show higher accuracy than neuropsychological evaluation alone regarding diagnosis of true AD.

In most of the mentioned PET-studies, the sensitivity of $[18 \mathrm{~F}] \mathrm{FDG}$ PET for detection of $\mathrm{AD}$ has been found to be very high (>90\%), whereas less high values have usually been reported for specificity. However, a number of studies demonstrated that automated procedures for observer-independent image evaluation may lead to a considerable improvement of this issue. A common approach consists in automated interindividual 3D-stereotactic spatial normalization of the image data, allowing voxel-based statistical comparison of patients data with data from healthy control subjects. Several programs for this type of analysis are available (e.g. Neurostat 3D-SSP, University of Michigan, USA or SPM, Wellcome Dept. London, GB). These programs facilitate the assessment of statistically significant abnormalities and potentially even the automated assignment to a diagnostic category. Their value in the diagnosis of AD has been extensively tested [8, $26,28,39,41,65]$. A large multicenter study ("NESTDD") was able to prove that the application of this type of voxel-based automated data-analyses to [18F]FDG PET data leads to an improved specificity of $93 \%$, while maintaining a sensitivity of $93 \%$, regarding the diagnosis of $\mathrm{AD}[39,41]$. With this approach, even in very mild dementia (at MMSE 24 or higher) sensitivity still reached $84 \%$ and specificity $93 \%$.

Another option for objective semiquantitative analysis of [18F]FDG PET data may be found in the application of regional ROI's. For this kind of approach, the patient's PET and MRI image data have often been coregistered at first in order to define anatomical ROI's on individual MRI data, which have been applied to the corresponding PET data, subsequently [22]. This type of approach, however, is time-consuming and requires manual ROI-definition. Data-analysis procedures allowing automated ROI-definition may be less susceptible for observer-dependent variations. Mosconi et al. introduced an automated and anatomically valid mask technique to sample the hippocampus on PET data (socalled HipMask). They were able to document hippocampal hypometabolism in DAT with this technique and to demonstrate that it may improve discrimination between healthy subjects, MCI and DAT-patients [69].

In summary, there is good evidence that diagnostic algorithms including [18F]FDG PET achieve better results than neuropsychological testing alone. It has been shown that [18F]FDG PET may actually be included into algorithms of dementia diagnostics in a cost- efficient way and that it indeed leads to an improvement of patient management, including therapy and care [75, 100,101]. So far, comparable results for most other imaging procedures are still pending. Of the existing MRI-techniques, MRI-based volume measurements of atrophy have been most detailed evaluated regarding the diagnostic value in detection of Alzheimer's disease $[50,55]$. Particularly, volumetry of the hippocampus appears to be able to differentiate between AD and healthy volunteers and some studies also document a correlation between in vivo MR-volumetry and post mortem neuropathological classification of AD [49]. However, the utility of these volumetric procedures to reliably classify the individual patient in a clinical setting has not been sufficiently verified and considerable scepticism about the added value of these procedures to standard clinical evaluation still remains [24,31,48,94, 113]. Alternative promising MR-imaging procedures, which may offer insights into various parameters of AD-pathology in the brain are also still in the process of validation [50].

So far, the number of studies directly comparing the accuracy of MRI-volumetry and [18F]FDG PET for diagnosis of $\mathrm{AD}$ is very limited. A small number of studies, however, indicate that [18F]FDG PET yields a better diagnostic performance for the discrimination between $\mathrm{AD}$ patients and healthy control subjects than does the morphologic assessment of atrophy, e.g. by voxel-based morphometry (VBM) [58]. Also a study by Kawachi and colleagues showed a slightly higher accuracy for detection of AD for [18F]FDG PET compared to VBM-MRI [51]. This impression is further confirmed by a number of studies demonstrating that the hypometabolic changes in measured by PET in AD exceed structural changes and are not representing an artefact due to an increase in CSF space induced by atrophy, but reflect a true metabolic reduction per gram of tissue $[11,46]$. Studies, which applied partial volume effect correction to eliminate the effects of atrophy on PET signal, consistently demonstrated that FDG uptake is reduced in AD regardless of whether or not partial volume effect correction is applied, supporting the notion that the reduced FDG uptake in these areas is not the result of atrophy. In a recent direct voxel-based comparison between gray matter hypometabolism and atrophy in Alzheimer's disease, Chetelat et al. were able to demonstrate that hypometabolism significantly exceeds atrophy in most altered structures, particularly in the posterior cingulate-precuneus, orbitofrontal, inferior temporo-parietal, parahippocampal, angular and fusiform areas in $\mathrm{AD}$ [17]. All these studies further sup- 
port the theory that functional changes advance major atrophy and, in consequence, functional imaging may be more sensitive to detect $\mathrm{AD}$ compared to structural imaging.

\section{3. [18F]FDG PET in MCI}

As mentioned above, following a period free of symptoms, it is assumed that patients go through a stage of so called mild cognitive impairment (MCI) for 5-10 years (often particularly in the memory domain), before clinically manifest dementia can be diagnosed [81-83]. Clinical diagnosis of MCI is defined by a loss of cognitive functions, which exceeds common age-associated changes but does not yet allow for the diagnosis of dementia (i.e. patients are not impaired regarding their activities of daily living) [83]. Thus, the MCI group is regarded as a risk population for $\mathrm{AD}$, and may be of particular interest regarding new treatment trials [34]. Consequently, also the current guidelines of the American Academy of Neurology recommend that patients with Mild Cognitive Impairment should be identified and monitored for progression to $\mathrm{AD}$, which is regarded to be a likely event [52].

So far, a number of studies have evaluated the value of $\left[{ }^{18} \mathrm{~F}\right]$ FDG PET in the diagnostic assessment of MCI. In several cross-sectional studies (some of them large multicenter studies with $>100$ MCI subjects), it has been consistently demonstrated that $\left[{ }^{18} \mathrm{~F}\right] \mathrm{FDG}$ PET imaging can reliably differentiate groups of MCIpatients from healthy controls and on the basis of specific hypometabolic patterns [23,26,27,66,74,76,78]. The most consistent abnormal finding, described in the majority of these studies is the hypometabolism in the posterior cingulate cortex. Depending on the study, this finding has been accompanied by more or less involvement of additional cortical regions affected by hypometabolism in manifest AD-dementia, such as the temporoparietal cortex and frontal cortical regions. As in $\mathrm{AD}$ occipital and sensorymotor cortical areas are spared from hypometabolic changes consistently.

There has been some controversy regarding the hypometabolic involvement of the medial temporal cortex, particularly of the hippocampus. Whereas some studies show hyometabolism in this region in MCI [20, 22,73 , others do not show major medial temporal hypometabolism in MCI or AD-dementia [6,27,40,63,66, 98]. The medial temporal cortex and in particular the hippocampus has often been discussed as a region in the brain early affected by pathological changes includ- ing atrophy in the course of AD. Given the fact that a major role in memory function has often been assigned to this brain structure, it seems reasonable to look for hypometabolism in this region in MCI. The fact that data on hypometabolic changes in MCI in this region are thereto limited may have two apparent reasons: first the assessment of cerebral hypometabolism in this region may be aggravated by the limited spatial resolution of PET. In fact, studies showing hypometabolism in the hippocampus predominantly applied specific data processing technologies, such as MR-based hippocampal ROI-definition or voxel-based analysis following correction of regional partial volume effects $[61,69]$. Thus, in other studies employing less specific approaches for the assessment of this region, this abnormality may have been missed. Another potential reason for the lack of hypometabolic findings in the hippocampus may be found in a regional compensatory effect, which has been increasingly discussed recently. The basic idea underlying this hypothesis is that a loss in the number of neurons in the hippocampus will be compensated by increased activity of the remaining neurons. Several studies support this view $[17,47,98]$. Samuraki et al. found reduced [18F]FDG uptake in posterior cingulate and parietal temporal lobe in mild $\mathrm{AD}$ but relatively preserved uptake in the MTL, including hippocampal areas, despite gray matter loss. These findings persisted even after partial volume correction. The authors suggested that compensatory mechanisms in the hippocampus may be responsible for these findings [98]. Similarly, in a recent study Chetelat et al. demonstrated that hypometabolism in the brain exceeds atrophy in several regions including the posterior cingulate, whereas less hypometabolism was found in the hippocampus compared to distinct atrophy in this region. Again, the authors suggested that compensatory mechanisms may be responsible for these findings [17].

Finally, some of the variance in the metabolic patterns detected in the MCI-group may be attributed to the type of criteria used for definition of MCI (amnestic, multiple domain versus single domain, etc.), to neuropsychological profiles [43,74], as well as to the wellknown heterogeneity of the MCI-group itself. Some MCI-subjects are suffering from diseases other than $\mathrm{AD}$, some will convert to dementia later than others and some may even get better over time and never develop dementia [82]. Thus, the most important diagnostic challenge is not to distinguish MCI-patients as a group from healthy controls, but rather the identification of those individual patients who will deteriorate to manifest dementia over time (i.e. those subject who 


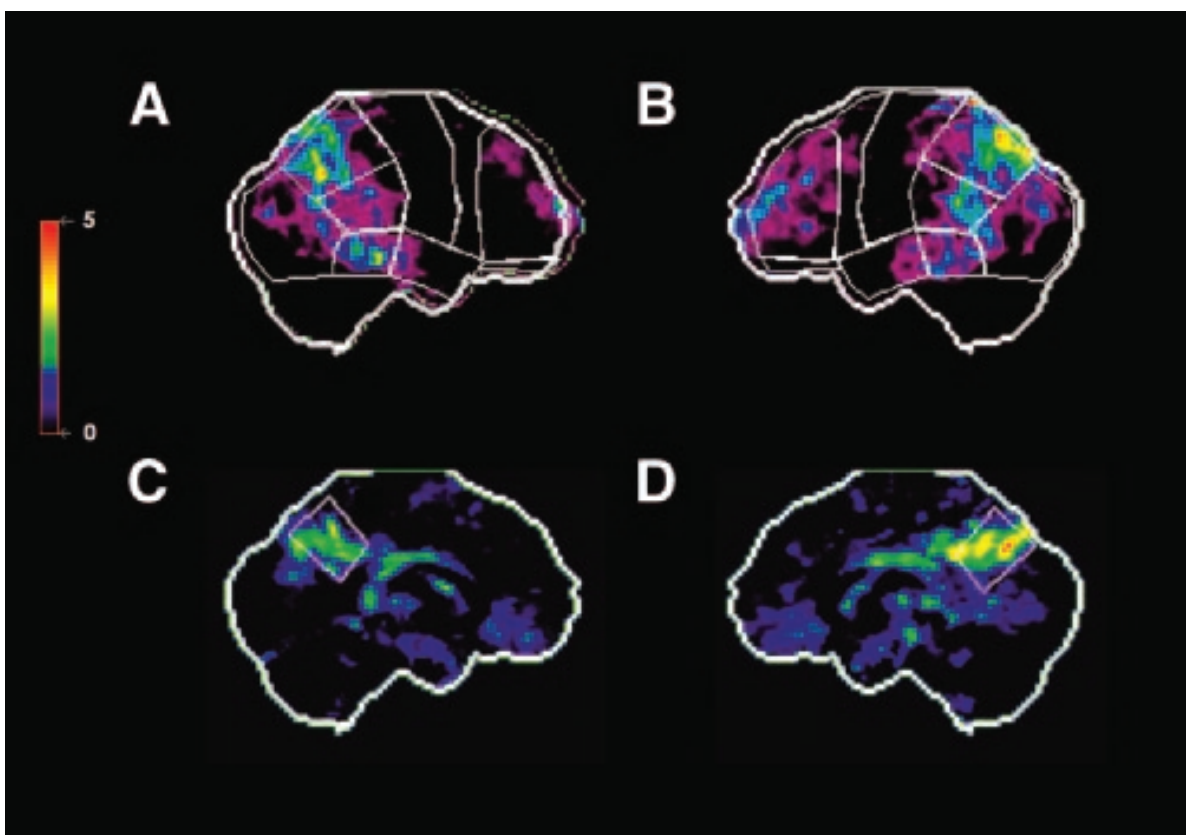

Fig. 2. Baseline [18F]FDG PET findings in a single MCI patient, who showed clinical progression to DAT within 16 months. Surface projections of statistical abnormalities (z-scores) as compared with a healthy control population are displayed. Predefined anatomic surface ROIs are depicted as white lines. Significant hypometabolism in bilateral temporoparietal and frontal cortex and in the posterior cingulate cortex is apparent. (A) Right lateral. (B) Left lateral. (C) Left medial. (D) Right medial. Reproduced with permission from Drzezga et al 2005 (Drzezga et al. 2005 ).

are actually suffering from AD). As discussed above, clinical/neuropsychological examination does not consistently permit a reliable prediction of conversion to dementia in the group of MCI-patients [5,83]. Thus a number of studies have been carried out to identify the predictive value of $[18 \mathrm{~F}] \mathrm{FDG}$ PET as a biomarker of ongoing AD in MCI [6,16,26,27,40,45,71,78,102]. Numbers of included patients in these studies ranged beween 17 and 55, mean follow-up periods ranged between 1-4 years and observed conversion rates ranged between $22-45 \%$. All of these studies were able to identify typical hypometabolic changes in [18F]FDG PET baseline examinations of MCI-patients, associated with later conversion to manifest $\mathrm{AD}$-dementia, whereas stable subjects showed less or no abnormalities. Again, most consistently across those studies, posterior cingulate hypometabolism was identified as a key finding, predicting later conversion of $\mathrm{MCI}$ to $\mathrm{AD}$, followed by parietotemporal and frontal hypometabolism. Generally high sensitivity and specificity values were calculated $(75 \%-100 \%)$ for [18F]FDG PET. The ultimate value of a diagnostic biomarker for predicting the clinical course can only be defined by comparison of baseline rating values against results from longitudinal follow up in the individual patient. A small number of studies tried to follow this longitudinal approach in
MCI. Silverman et al included a subset of 55 patients with only mild cognitive impairment in their longitudinal multi-center study. They were able to demonstrate that progression of cognitive deterioration was predictable on the basis of baseline PET-findings with a sensitivity of $91 \%$ and $75 \%$ specificity in this sample [102]. A smaller study employing automated image analysis and classification of patients according to predefined criteria at baseline was able to achieve a sensitivity of $92 \%$ and a specificity of $89 \%$ (positive predictive value $85 \%$ negative predictive value $94 \%$ ) regarding conversion to AD 16 months later (see Fig. 2) [26].

In MCI, correlation of imaging data with post mortem data has not been frequently performed. Two studies demonstrate that [18F]FDG has a high sensitivity regarding identification of actual ongoing AD in the stage of MCI or even in cognitively healthy subjects, as verified by post mortem assessment years after the original PET examination [70,102]. Mosconi et al did also demonstrate that PET based diagnosis remained consistent in repeated measurements in the same subjects over longer time periods. Silverman and colleagues demonstrated in their multi-center study that [18F]FDG PET was able to detect ongoing Alzheimer's disease in subjects with mild cognitive symptoms as verified later by post mortem neuropathological assessment with $95 \%$ sensitivity and $71 \%$ specificity [102]. 
Again, as for DAT, it has been shown also for MCI that automated data-analysis procedures minimizing observer-influence may considerably improve image assessment. Particularly the automated individual comparison of patient data with healthy control populations shows promising results $[27,28,61,66,74]$. In a recent study it has been demonstrated that the careful selection of a longitudinally controlled truly healthy control population may be crucial for the quality of the results gained from this type of study [74]. Furthermore, it has been shown that the selection of the region used for normalization of the individual data may influence the sensitivity of the examination [115].

Regarding this array of promising results, it is not surprising that several studies were able to demonstrate higher accuracy of [18F]FDG for prediction of ADdementia in MCI-patients, compared to neuropsychological examination [71,102]. Even when post-mortem neuropathological evaluation was considered the goldstandard, it was obvious that adding [18F]FDG to the diagnostic setup did improve prediction accuracy [102]. In contrast to these findings, there is considerably more controversy regarding the added value of volumetric MRI examinations regarding early identification of AD. Currently, volumetric assessment of the medial temporal lobe/hippocampus can be regarded the best evaluated parameter regarding the identification of AD in early stages of the disease $[4,18,24,29,54,109,112]$. However, the number of studies reporting on the actual predictive value of these measurements in longitudinal studies is relatively limited and the actually reported predictive values are rather low. E.g. Korf et al reported a sensitivity of $70 \%$ and a specificity of $68 \%$ (positive predictive value $68 \%$, negative predictive value $70 \%$ ) for visually assessed medial temporal lobe atrophy [54]. Devanand demonstrated sensitivity and specificity of $67 \%$ : and $80 \%$, respectively for measurements of hippocampus and entorhinal cortex [24]. They conclude that the added value of MR imaging to clinical parameters such as age and cognitive variables remains rather limited, as far as prediction of clinical course is concerned. Also Fleisher et al. in their recent study summarize that in moderate stages of amnestic MCI, common cognitive tests provide better predictive accuracy than MRI measures of whole brain, ventricular, entorhinal cortex, or hippocampal volumes for assessing progression to DAT [31].

Some studies imply that longitudinal assessment of atrophy rates in the hippocampus and possibly also even of the whole brain may be more sensitive regarding future cognitive deterioration, and thus may have predictive value in early stages such as MCI [103, 106]. However, e.g. Jack et al. discussed in their study that the considerable overlap between converters and non-converters indicates that these measures are unlikely to provide absolute prognostic information for individual patients [48]. Furthermore concerns have been raised regarding the clinical practicability for serial MRI imaging regarding increased costs, complexity of serial image coregistration and the clinical need to promptly establish the diagnosis [24]. It may also be mentioned in this context that a recent post mortem study correlating post mortem MRI-measurements of medial temporal lobe atrophy (MTA) with specific neuropathology demonstrated that MTA is neither specific nor does it show a very high sensitivity (63\%) for AD [7].

In correspondence to these findings and interpretations, studies on direct comparison of the diagnostic value of [18F]FDG PET and MRI-methods in MCI predominantly report relatively higher accuracy of PET. As an example, De Santi et al. demonstrated that [18F]FDG PET measurements achieved higher accuracy compared to MRI volume measurements regarding diagnostic classification of MCI [22]. Also Yuan et al. recently demonstrated that [18F]FDG PET can be regarded superior to MRI in predicting conversion in MCI [116]. These findings correspond well to the hypothesis that functional changes appear earlier in the history of AD and that hypometabolism advances and exceeds atrophy and is better suited for early diagnosis $[58,72,98]$.

Apart from volumetric assessment of regional brain atrophy, various other modern MRI-techniques may allow to assess different aspects of Alzheimer's disease pathology, such as diffusion weighted imaging, perfusion imaging and arterial spin labelling, assessment of resting state activity and spectroscopy. Most of these techniques, however, have only been studied in small samples and information on their value for early diagnosis of $\mathrm{AD}$ is yet limited. Their predictive value regarding conversion from $\mathrm{MCI}$ to $\mathrm{AD}$ in large cohorts remains to be established [24,50].

Consequently, the recent guidelines (2001, reaffirmed 2004) for dementia diagnosis of the American Academy of Neurology (AAN) explicitly state that linear or volumetric MR or CT measurement strategies should not be included in the routine evaluation of the demented patient. It is concluded that measurement of hippocampal atrophy by MRI may not be useful in clinical practice because of its low precision. It is also discussed that [18F]FDG-PET appears superi- 


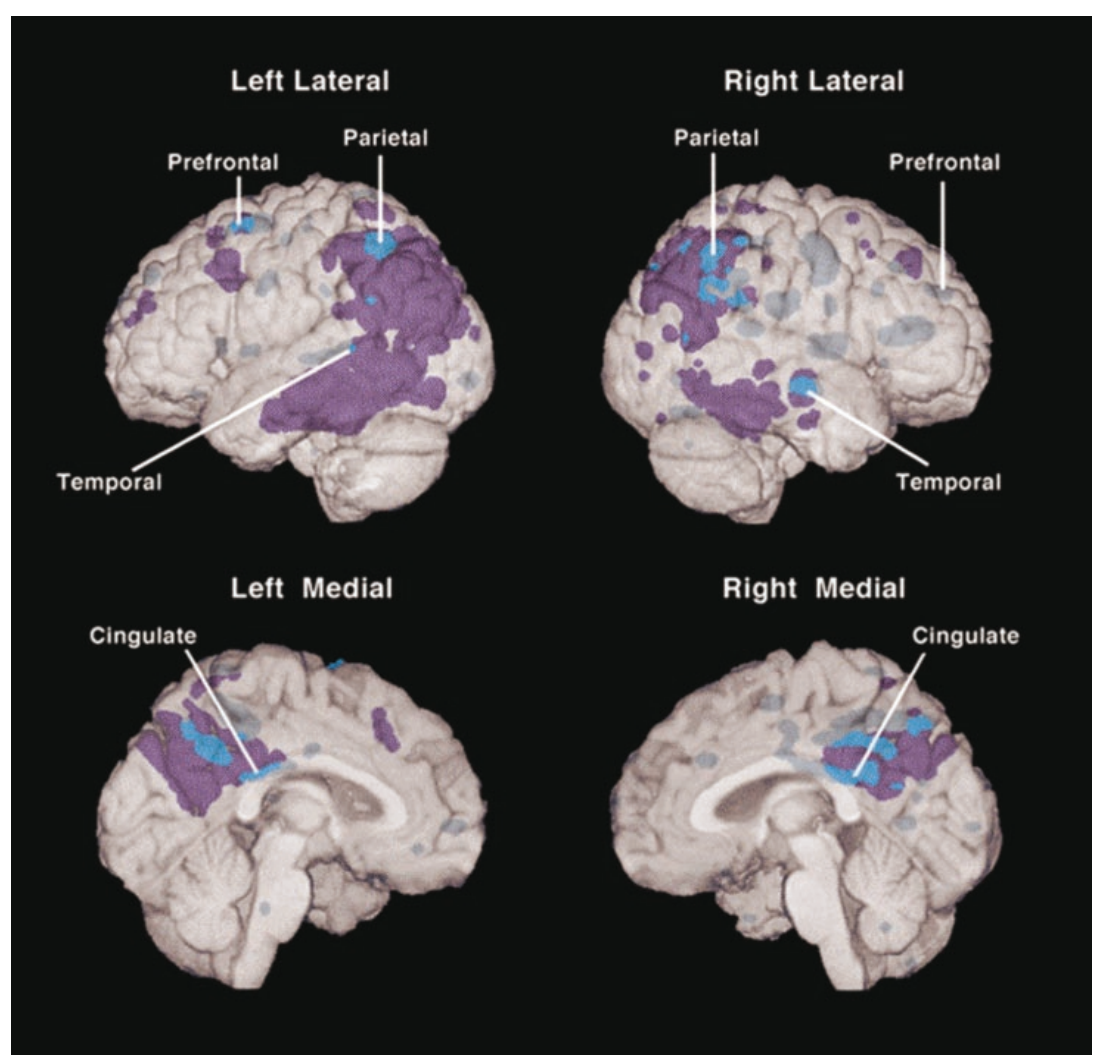

Fig. 3. Regions of the brain with abnormally low CMRgl in young adult carriers of the APOE e4-allele and their relation to brain regions with abnormally low CMRgl in patients with probable DAT. The dark (purple in coloured online version) are regions in which CMRgl was abnormally low only in the patients with DAT, the bright (bright blue in the coloured online version) areas are regions in which CMRgl was abnormally low in both the young adult e 4 carriers and patients with probable DAT, and the grey areas (muted blue in the coloured online version) are regions in which CMRgl was abnormally low only in the e4 carriers. (Lines point to the locations of the e4 carriers' most significant CMRgl reductions. Reproduced with permission from Reiman et al. 2004 (Reiman et al. 2004) (C) 2003 by The National Academy of Sciences of the USA.

or to MRI measures of hippocampal atrophy because changes in cerebral glucose metabolism antedate the onset of memory decline, whereas the MRI hippocampal changes do not. Thus, it is concluded in these guidelines that PET scanning appears to have promise for use as an adjunct to clinical diagnosis, but further prospective studies with PET are needed to establish its added value over a competent clinical diagnosis [52]. Interestingly in these guidelines the evaluation and clinical monitoring of persons with MCI is explicitly recommended, due to their increased risk for developing dementia [29,53]. However, no detailed discussion on the value of imaging biomarkers in the stage of MCI is provided. These guidelines date back to 2001 and since then abundant literature has been added, particularly on the value $[18 \mathrm{~F}]$ FDG PET for early diagnosis of AD in MCI. Thus, it may be considered to include this aspect in upcoming versions of these guidelines.

When using [18F]FDG PET for diagnosis in MCI and $\mathrm{AD}$, it needs to be kept in mind that the degree of cognitive impairment will not necessarily correlate linearly with the extent of cerebral hypometabolism in each subject. A number of studies were able to demonstrate that subjects who were expected to have higher cognitive reserve, i.e. due to higher levels of education, showed more pronounced hypometabolism as compared to subjects with lower levels of education and similar degree of cognitive impairment [80,107]. Recently, a study was able to demonstrate that this type of cognitive reserve effects may also be observed in MCI subjects [35]. These cognitive reserve effects further underline that a diagnosis based on cognitive symptoms alone may not be suitable to assess the extent of damage in the brain.

\section{4. [18F]FDG PET in asymptomatic risk persons}

There is common agreement that the brain pathology of $\mathrm{AD}$ starts years before onset of even mild clinical 
symptoms, i.e. even ahead of the stage of MCI. In principle, it may make sense to start new therapeutic strategies even in presymptomatic stages, to avoid neuronal damage before it leads to cognitive impairment. Thus, the identification of biomarkers allowing the diagnosis of $\mathrm{AD}$ in asymptomatic stages appears to be a worthwhile aim. According to current diagnostic guidelines, there are still insufficient data to make any recommendations regarding cognitive screening of asymptomatic individuals [82]. Thus, in a first step it appears reasonable to focus on healthy subjects, who bear an increased risk for developing AD-type dementia later in life, i.e. they potentially may suffer already from ongoing neuropathology, even without apparent cognitive symptoms. Apparently, subjects carrying genes encoding for the familiar forms of Alzheimer's diseases represent such a risk population. In fact one study was able to demonstrate hypometabolic abnormalities in subjects carrying mutations in the Presenilin 1 gene, responsible for early-onset autosomal dominant $\mathrm{AD}$ (FAD) [72]. Presymptomatic FAD individuals showed hypometabolic changes in [18F]FDG PET scans, consistent with the typical AD PET pattern in the relative absence of structural brain atrophy. The authors concluded that these data further suggest that [18F]FDG PET measures may serve as biomarkers for the preclinical diagnosis of AD. However, the familiar cases of autosomal dominant inherited $\mathrm{AD}$ only represent a small percentage of all AD-cases and they are different regarding onset and clinical course compared to the frequent sporadic form. Thus, findings obtained in this population may not be easily transferred to the general population of sporadic AD. Apart from autosomal dominant forms of $\mathrm{AD}$, a higher risk for sporadic $\mathrm{AD}$ may be defined by familiar disposition (positive familiy history for AD), or by known factors of genetic susceptibility such as the ApoE e4-positive genotype. The ApoE e4-allele represents the greatest known risk factor for sporadic $A D$, increasing the relative risk to suffer from AD for about 3-15 times [33], depending on the e4allele frequency. Correspondingly, several studies have been able to demonstrate hypometabolic abnormalities in healthy carriers of the ApoE e4-genotype compared to e4-negative controls. For example, Reiman et al showed reduced glucose metabolism in e4-positive subjects in the same regions of the brain as in patients with probable Alzheimer's disease (posterior cingulate, parietal, temporal, and prefrontal regions) [91]. Members of the same group later demonstrated that even relatively young (20-39 years) e4-carriers had abnormally low rates of glucose metabolism bilaterally in the posterior cingulate, parietal, temporal, and prefrontal cortex and that the e4-gene dose is correlated with lower glucose metabolism in each of these brain regions (see Fig. 3) [92,93]. Furthermore in several studies specific decline of glucose metabolism over time in the AD-typical regions has been demonstrated in cognitively healthy e4-carriers $[90,105]$. Correspondingly, more pronounced hypometabolism was detected in e4positive subjects with manifest DAT compared to agematched e4-negative DAT-patients [31]. Conclusively, it has been suggested that brain imaging techniques could be used to evaluate putative $\mathrm{AD}$ prevention therapies in cognitively normal $A p o E$ epsilon4 carriers and that they could help guide the investigation of a molecular mechanism associated with AD vulnerability [89]. Apart from subjects with ApoE e4-positive genotype, another very recent study was also able to demonstrate similar hypometabolic changes in subjects with maternal history of $\mathrm{AD}$, who are also at higher risk for dementia [70].

However, both family history and the ApoE genotype do only indicate a theoretical risk and are no proof for the actual presence of a neurodegenerative disorder. Many carriers of the 4-allele will never develop DAT. This implies that significant abnormalities were detected in the mentioned studies in the risk populations when compared with healthy controls, although the risk-groups will most probably have contained several subjects who were not suffering from ongoing neurodegeneration. Thus, it appears particularly interesting to evaluate the value of these detected abnormalities, regarding the prediction of cognitive decline in individual subjects in the future. In a single [18F]FDG PET study De Leon et al. were able to demonstrate that baseline metabolic reductions in the entorhinal cortex of healthy subjects accurately predicted the conversion from normal to MCI within a 3-year follow up period [23]. In the subjects who declined, the baseline entorhinal metabolism predicted longitudinal memory and temporal neocortical metabolic reductions. More data needs to be collected in larger longitudinal studies over long time intervals, to evaluate the utility of [18F]FDG PET for individual prognosis/early detection of $\mathrm{AD}$ in healthy subjects and its actual value as a screening tool.

\section{Multi-modal approaches}

It is commonly assumed that AD represents a complex multifactorial disorder, which will not necessari- 
ly follow the identical course in every single patient. Thus, in general the most appropriate way to ensure reliable early diagnosis of AD may be found in the combination of several diagnostic tools, focussing on different aspects of the disease. This includes clinical and neuropsychological testing as well as different types of biomarkers such as the assessment of genotype, cerebrospinal fluid markers and functional/molecular and structural imaging procedures. A number of studies suggest superior performance of diagnostic setups combining different diagnostic markers for detection of $\mathrm{AD}$ in the stage of MCI. This includes studies on the combination of imaging and non-imaging biomarkers [24, $29,68,71,105,111]$, as well as on the combination of different imaging modalities $[27,51,114]$. Two studies were able to demonstrate that the combination of the ApoE-genotype, encoding for AD-susceptibility, with [18F]FDG-PET as a marker of actual brain pathology, could significantly improve either sensitivity or specificity of DAT-prediction in MCI-patients [29,71]. Also the current AAN guidelines suggest that combining medial temporal measures with other markers, such as functional neuroimaging or ApoE-genotyping, may improve diagnostic accuracy [52].

The discussion about combining different diagnostic entities to find to a comprehensive diagnosis may gain even more momentum in the future, regarding two recent developments. One being new PET tracers for molecular imaging of amyloid plaque deposition such as [11C]PIB [28,79,96]. First studies suggest a high predictive value of this type of tracer for prediction of $\mathrm{AD}$ in MCI [35] and a small study suggests superior classification of AD compared to [18F]FDG-PET [77]. However, the relatively high numbers of PIB-positives in older asymptomatic subjects require further longitudinal studies [67]. [18F]FDG-PET may be highly valuable to detect the onset of actual functional pathology in PIB-positive subjects. Thus, PIB-PET may serve as a risk-marker, whereas FDG-PET may denote the actual onset of disease. A second important development lies in the current efforts to establish a new hybrid instrument, combining PET and MR imaging technology into one single scanner. This hybrid imaging technology may significantly influence diagnostic protocols, as it will allow simultaneous acquisition of functional, molecular and structural information in one session in the future $[17,18]$.

\section{Conclusion}

In conclusion, imaging of the cerebral glucose metabolism with [18F]FDG-PET as a marker of neu- ronal function can be regarded as a highly sensitive and specific tool for early and accurate diagnosis of Alzheimer's disease. It has been demonstrated even by post mortem neuropathological assessment that this imaging technique shows better diagnostic performance than exclusive neuropsychological evaluation. Furthermore excellent performance of [18F]FDG for early diagnosis of this disorder even in early mild symptomatic stages (MCI) has been demonstrated, i.e. before a diagnosis of dementia is possible by neuropsychological assessment. The documentation of abnormal findings in risk persons even in asymptomatic stages underlines the very high sensitivity of this tool. Current data indicate that [18F]FDG PET shows superior performance regarding prediction of cognitive decline in MCI, compared to established structural imaging methods, such as MR-volumetry. These findings are corresponding to the hypothesis that functional changes occur prior to structural changes in the brain. Current guidelines on dementia diagnostics discuss a potential role of [18F]FDG imaging for diagnosis of dementia and discuss its superiority compared to MR-volumetry, but they do not explicitly recommend the performance of [18F]FDG PET. However, the role of imaging procedures for diagnosis of AD in stages ahead of manifest dementia such as MCI is not discussed in these guidelines, although careful diagnostic evaluation of MCI-patients, representing a high-risk group for dementia, is strongly advised. Regarding the fact that AD-pathology starts years to decades prior to the onset of clinically manifest dementia, it becomes obvious that diagnostic efforts should be directed towards diagnosis of Alzheimer's "Disease" rather than of Alzheimer's "Dementia". Neuronal dysfunction, which can be detected with [18F]FDG PET may be regarded as the actual onset of Alzheimer's disease in the brain, disregarding symptomatic stage. In this context and regarding the abundant amount of new information on the value of [18F]FDG PET, which has been collected in the recent years, it may be considered to include [18F]FDG -PET as a highly sensitive marker of disease in early stages in the current guidelines for diagnosis of "dementia”. Apart from the value of [18F]FDG PET alone, certain combinations of this imaging marker with other biomarkers such as genotype or CSF-markers may offer promising approaches to assess individual prognosis in AD-patients. New developments in the field of imaging, such as tracers for amyloid plaque imaging, modern MR-procedures and new hybrid imaging technologies such as PET-MR may add further material to this discussion. In the future, these exciting options may 
offer the opportunity to address a number of different diagnostic questions, e.g.: a) the detection of protein deposition pathology with amyloid plaque imaging, b) the onset of neuronal dysfunction (i.e. the beginning of actual Alzheimer's disease) with [18F]FDG and c) the presence of brain atrophy and other structural and biochemical consequences of the disease with MRI.

It can be summarized that the value of [18F]FDG for early diagnosis of $\mathrm{AD}$ has been systematically proven. At present, comparable qualities regarding sensitivity, specificity, value for therapy control and even costefficiency in AD have not been documented for other imaging procedures. Therefore, PET can be regarded as a method of choice regarding diagnosis of ongoing AD-neurodegeneration, particularly in patients with mild cognitive impairment.

\section{Acknowledgment}

This work has been supported in part by DFG-grants (Deutsche Forschungsgemeinschaft) Project Numbers: DR 445/3-1, DR 445/4-1 (Drzezga). The author thanks Nicola Karina for the careful review of the manuscript.

\section{References}

[1] Alzheimer's disease facts and figures 2008, Alzheimers Dement 4(2), 110-133.

[2] H. Aguero-Torres, B. Winblad and L. Fratiglioni, Epidemiology of vascular dementia: some results despite research limitations, Alzheimer Dis Assoc Disord 13(Suppl 3) (1999), S15-S20.

[3] O. Almkvist and B. Winblad, Early diagnosis of Alzheimer dementia based on clinical and biological factors, European Archives of Psychiatry \& Clinical Neuroscience 249(Suppl 3) (1999), 3-9.

[4] L.G. Apostolova, R.A. Dutton, I.D. Dinov et al., Conversion of mild cognitive impairment to Alzheimer disease predicted by hippocampal atrophy maps, Arch Neurol 63(5) (2006), 693-699.

[5] E. Arnaiz, O. Almkvist, R.J. Ivnik et al., Mild cognitive impairment: a cross-national comparison, J Neurol Neurosurg Psychiatry 75(9) (2004), 1275-1280.

[6] E. Arnaiz, V. Jelic, O. Almkvist et al., Impaired cerebral glucose metabolism and cognitive functioning predict deterioration in mild cognitive impairment, Neuroreport 12(4) (2001), 851-855.

[7] F. Barkhof, T.M. Polvikoski, E.C. van Straaten et al., The significance of medial temporal lobe atrophy: a postmortem MRI study in the very old, Neurology 69(15) (2007), 15211527.

[8] P. Bartenstein, S. Minoshima, C. Hirsch et al., Quantitative assessment of cerebral blood flow in patients with Alzheimer's disease by SPECT, J Nucl Med 38(7) (1997), 1095-1101.
[9] H. Bickel, [Dementia syndrome and Alzheimer disease: an assessment of morbidity and annual incidence in Germany], Gesundheitswesen 62(4) (2000), 211-218.

[10] K. Blennow, M.J. de Leon and H. Zetterberg, Alzheimer's disease, Lancet 368(9533) (2006), 387-403.

[11] A.L. Bokde, P. Pietrini, V. Ibanez et al., The effect of brain atrophy on cerebral hypometabolism in the visual variant of Alzheimer disease, Arch Neurol 58(3) (2001), 480-486.

[12] E. Braak, K. Griffing, K. Arai et al., Neuropathology of Alzheimer's disease: what is new since A. Alzheimer, Eur Arch Psychiatry Clin Neurosci 249(Suppl 3) (1999), 14-22.

[13] H. Braak and E. Braak, Neuropathological stageing of Alzheimer-related changes, Acta Neuropathol (Berl) 82(4) (1991), 239-259.

[14] C. Catana, Y. Wu, M.S. Judenhofer et al., Simultaneous acquisition of multislice PET and MR images: initial results with a MR-compatible PET scanner, J Nucl Med 47(12) (2006), 1968-1976.

[15] C. Catana, S.R. Cherry and A.G. Sorensen, Combined Positron Emission Tomography and Magnetic Resonance Imaging Scanners - Potential Neurological Applications, US Neurology 4(2) (2008), 76-80.

[16] G. Chetelat, B. Desgranges, V. de la Sayette et al., Mild cognitive impairment: Can FDG-PET predict who is to rapidly convert to Alzheimer's disease, Neurology 60(8) (2003), 1374-1377.

[17] G. Chetelat, B. Desgranges, B. Landeau et al., Direct voxel based comparison between grey matter hypometabolism and atrophy in Alzheimer's disease, Brain 131(Pt 1) (2008), 6071.

[18] G. Chetelat, B. Landeau, F. Eustache et al., Using voxel based morphometry to map the structural changes associated with rapid conversion in MCI: A longitudinal MRI study, Neuroimage (2005).

[19] L. Davies, B. Wolska, C. Hilbich et al., A4 amyloid protein deposition and the diagnosis of Alzheimer's disease: prevalence in aged brains determined by immunocytochemistry compared with conventional neuropathologic techniques, Neurology 38(11) (1988), 1688-1693.

[20] M.J. de Leon, A. Convit, O.T. Wolf et al., Prediction of cognitive decline in normal elderly subjects with 2[(18)F]fluoro-2-deoxy-D-glucose/poitron -emission tomography (FDG/PET), Proc Natl Acad Sci USA 98(19) (2001), 10966-10971.

[21] M.J. de Leon, L. Mosconi, K. Blennow et al., Imaging and CSF studies in the preclinical diagnosis of Alzheimer's disease, Ann N Y Acad Sci 1097 (2007), 114-145.

[22] S. De Santi, M.J. de Leon, H. Rusinek et al., Hippocampal formation glucose metabolism and volume losses in MCI and AD, Neurobiol Aging 22(4) (2001), 529-539.

[23] A. Del Sole, F. Clerici, A. Chiti et al., Individual cerebral metabolic deficits in Alzheimer's disease and amnestic mild cognitive impairment: an FDG PET study, Eur J Nucl Med Mol Imaging 35(7) (2008), 1357-1366.

[24] D.P. Devanand, G. Pradhaban, X. Liu et al., Hippocampal and entorhinal atrophy in mild cognitive impairment: prediction of Alzheimer disease, Neurology 68(11) (2007), 828-836.

[25] A. Drzezga, T. Grimmer, G. Henriksen et al., Imaging of amyloid plaques and cerebral glucose metabolism in semantic dementia and Alzheimer's disease, Neuroimage 39(2) (2008), 619-633.

[26] A. Drzezga, T. Grimmer, M. Riemenschneider et al., Prediction of individual clinical outcome in MCI by means of 
genetic assessment and (18)F-FDG PET, $J$ Nucl Med 46(10) (2005), 1625-1632.

[27] A. Drzezga, N. Lautenschlager, H. Siebner et al., Cerebral metabolic changes accompanying conversion of mild cognitive impairment into Alzheimer's disease: a PET follow-up study, Eur J Nucl Med Mol Imaging 30(8) (2003), 11041113.

[28] A. Drzezga, M. Riemenschneider, B. Strassner et al., Cerebral glucose metabolism in patients with $\mathrm{AD}$ and different ApoE genotypes, Neurology 64(1) (2005), 102-107.

[29] R. Duara, D.A. Loewenstein, E. Potter et al., Medial temporal lobe atrophy on MRI scans and the diagnosis of Alzheimer disease, Neurology 71(24) (2008), 1986-1992.

[30] L.A. Farrer, L.A. Cupples, J.L. Haines et al., Effects of age, sex, and ethnicity on the association between apolipoprotein E genotype and Alzheimer disease. A meta-analysis. ApoE and Alzheimer Disease Meta Analysis Consortium, JAMA 278(16) (1997), 1349-1356.

[31] A.S. Fleisher, S. Sun, C. Taylor et al., Volumetric MRI vs clinical predictors of Alzheimer disease in mild cognitive impairment, Neurology 70(3) (2008), 191-199.

[32] A. Forsberg, H. Engler, O. Almkvist et al., PET imaging of amyloid deposition in patients with mild cognitive impairment. Neurobiol Aging, Oct 29(10) (2007), 1456-1465.

[33] H. Forstl and A. Kurz, Clinical features of Alzheimer's disease, Eur Arch Psychiatry Clin Neurosci 249(6) (1999),288290.

[34] T. Fuchsberger, F. Padberg, F. Faltraco et al., [Starting Alzheimer therapy in early stages whenever possible. Activities of daily living remain intact longer], MMW Fortschr Med 144(20) (2002), 36-39.

[35] V. Garibotto, B. Borroni, E. Kalbe et al., Education and occupation as proxies for reserve in aMCI converters and AD: FDG-PET evidence, Neurology 71(17) (2008), 1342-1349.

[36] J.A. Hardy and G.A. Higgins, Alzheimer's disease: the amyloid cascade hypothesis, Science 256(5054) (1992), 184-185.

[37] L.E. Hebert, P.A. Scherr, J.L. Bienias et al., Alzheimer disease in the US population: prevalence estimates using the 2000 census, Arch Neurol 60(8) (2003), 1119-1122.

[38] K. Herholz, FDG PET and differential diagnosis of dementia, Alzheimer Dis Assoc Disord 9(1) (1995), 6-16.

[39] K. Herholz, PET studies in dementia, Ann Nucl Med 17(2) (2003), 79-89.

[40] K. Herholz, A. Nordberg, E. Salmon et al., Impairment of neocortical metabolism predicts progression in Alzheimer's disease, Dement Geriatr Cogn Disord 10(6) (1999), 494504.

[41] K. Herholz, E. Salmon, D. Perani et al., Discrimination between Alzheimer dementia and controls by automated analysis of multicenter FDG PET, Neuroimage 17(1) (2002), 302316.

[42] J.M. Hoffman, K.A. Welsh-Bohmer, M. Hanson et al., FDG PET imaging in patients with pathologically verified dementia, J Nucl Med 41(11) (2000), 1920-1928.

[43] C. Huang, P. Mattis, K. Perrine et al., Metabolic abnormalities associated with mild cognitive impairment in Parkinson disease, Neurology 70(16 Pt 2) (2008), 1470-1477.

[44] M. Hull, M. Berger and M. Heneka. Disease-modifying therapies in Alzheimer's disease: how far have we come, Drugs 66(16) (2006), 2075-2093.

[45] A. Hunt, P. Schonknecht, M. Henze et al., Reduced cerebral glucose metabolism in patients at risk for Alzheimer's disease, Psychiatry Res 155(2) (2007), 147-154.
[46] V. Ibanez, P. Pietrini, G.E. Alexander et al., Regional glucose metabolic abnormalities are not the result of atrophy in Alzheimer's disease, Neurology 50(6) (1998), 1585-1593.

[47] K. Ishii, M. Sasaki, S. Yamaji et al., Relatively preserved hippocampal glucose metabolism in mild Alzheimer's disease, Dement Geriatr Cogn Disord 9(6) (1998), 317-322.

[48] C.R. Jack, Jr., M.M. Shiung, S.D. Weigand et al., Brain atrophy rates predict subsequent clinical conversion in normal elderly and amnestic MCI, Neurology 65(8) (2005), 12271231.

[49] W.J. Jagust, L. Zheng, D.J. Harvey et al., Neuropathological basis of magnetic resonance images in aging and dementia, Ann Neurol 63(1) (2008), 72-80.

[50] K. Kantarci and C.R. Jack, Jr., Quantitative magnetic resonance techniques as surrogate markers of Alzheimer's disease, NeuroRx 1(2) (2004), 196-205.

[51] T. Kawachi, K. Ishii, S. Sakamoto et al., Comparison of the diagnostic performance of FDG -PET and VBM -MRI in very mild Alzheimer's disease, Eur J Nucl Med Mol Imaging 33(7) (2006), 801-809.

[52] D.S. Knopman, S.T. DeKosky, J.L. Cummings et al., Practice parameter: diagnosis of dementia (an evidence-based review). Report of the Quality Standards Subcommittee of the American Academy of Neurology, Neurology 56(9) (2001), 1143-1153.

[53] D.S. Knopman, J.E. Parisi, A. Salviati et al., Neuropathology of cognitively normal elderly, J Neuropathol Exp Neurol 62(11) (2003), 1087-1195.

[54] E.S. Korf, L.O. Wahlund, P.J. Visser et al., Medial temporal lobe atrophy on MRI predicts dementia in patients with mild cognitive impairment, Neurology 63(1) (2004), 94-100.

[55] S. Lehericy, M. Marjanska, L. Mesrob et al., Magnetic resonance imaging of Alzheimer's disease, Eur Radiol 17(2) (2007), 347-362.

[56] A. Lim, D. Tsuang, W. Kukull et al., Clinico neuropathological correlation of Alzheimer's disease in a community -based case series, J Am Geriatr Soc 47(5) (1999), 564-569.

[57] P.J. Magistretti and L. Pellerin. Cellular mechanisms of brain energy metabolism and their relevance to functional brain imaging, Philos Trans $R$ Soc Lond B Biol Sci 354(1387) (1999), 1155-1163.

[58] I. Matsunari, M. Samuraki, W.P. Chen et al., Comparison of 18F-FDG PET and optimized voxel-based morphometry for detection of Alzheimer's disease: aging effect on diagnostic performance, J Nucl Med 48(12) (2007), 1961-1970.

[59] J.C. Mazziotta, R.S. Frackowiak and M.E. Phelps. The use of positron emission tomography in the clinical assessment of dementia, Semin Nucl Med 22(4) (1992), 233-246.

[60] G. McKhann, D. Drachman, M. Folstein et al., Clinical diagnosis of Alzheimer's disease: report of the NINCDS ADRDA Work Group under the auspices of Department of Health and Human Services Task Force on Alzheimer's Disease, Neurology 34(7) (1984), 939-944.

[61] K. Mevel, B. Desgranges, J.C. Baron et al., Detecting hippocampal hypometabolism in Mild Cognitive Impairment using automatic voxel -based approaches, Neuroimage 37(1) (2007), 18-25.

[62] S. Minoshima, Imaging Alzheimer's disease: clinical applications, Neuroimaging Clin N Am 13(4) (2003), 769-780.

[63] S. Minoshima, N.L. Foster and D.E. Kuhl, Posterior cingulate cortex in Alzheimer's disease, Lancet 344(8926) (1994), 895.

[64] S. Minoshima, N.L. Foster, A.A. Sima et al., Alzheimer's disease versus dementia with Lewy bodies: cerebral metabol- 
ic distinction with autopsy confirmation, Ann Neurol 50(3) (2001), 358-365.

[65] S. Minoshima, K.A. Frey, R.A. Koeppe et al., A diagnostic approach in Alzheimer's disease using three-dimensional stereotactic surface projections of fluorine-18-FDG PET, $J$ Nucl Med 36(7) (1995), 1238-1248.

[66] S. Minoshima, B. Giordani, S. Berent et al., Metabolic reduction in the posterior cingulate cortex in very early Alzheimer's disease, Ann Neurol 42(1) (1997), 85-94.

[67] M.A. Mintun, G.N. Larossa, Y.I. Sheline et al., [11C]PIB in a nondemented population: potential antecedent marker of Alzheimer disease, Neurology 67(3) (2006), 446-452.

[68] P.J. Modrego, Predictors of conversion to dementia of probable Alzheimer type in patients with mild cognitive impairment, Curr Alzheimer Res 3(2) (2006), 161-170.

[69] L. Mosconi, Brain glucose metabolism in the early and specific diagnosis of Alzheimer's disease. FDG-PET studies in MCI and AD, Eur J Nucl Med Mol Imaging 32(4) (2005), 486-510.

[70] L. Mosconi, R. Mistur, R. Switalski et al., FDG-PET changes in brain glucose metabolism from normal cognition to pathologically verified Alzheimer's disease, Eur J Nucl Med Mol Imaging (2009).

[71] L. Mosconi, D. Perani, S. Sorbi et al., MCI conversion to dementia and the $A p o E$ genotype: a prediction study with FDG -PET, Neurology 63(12) (2004), 2332-2340.

[72] L. Mosconi, S. Sorbi, M.J. de Leon et al., Hypometabolism exceeds atrophy in presymptomatic early-onset familial Alzheimer's disease, J Nucl Med 47(11) (2006), 1778-1786.

[73] L. Mosconi, W.H. Tsui, S. De Santi et al., Reduced hippocampal metabolism in MCI and AD: automated FDG-PET image analysis, Neurology 64(11) (2005), 1860-1867.

[74] L. Mosconi, W.H. Tsui, K. Herholz et al., Multicenter standardized 18F-FDG PET diagnosis of mild cognitive impairment, Alzheimer's disease, and other dementias, J Nucl Med 49(3) (2008), 390-398.

[75] G. Moulin -Romsee, A. Maes, D. Silverman et al., Cost effectiveness of $18 \mathrm{~F}$-fluorodeoxyglucose positron emission tomography in the assessment of early dementia from a Belgian and European perspective, Eur J Neurol 12(4) (2005), 254-263.

[76] P.J. Nestor, T.D. Fryer, P. Smielewski et al., Limbic hypometabolism in Alzheimer's disease and mild cognitive impairment, Ann Neurol 54(3) (2003), 343-351.

[77] S. Ng, V.L. Villemagne, S. Berlangieri et al., Visual assessment versus quantitative assessment of $11 \mathrm{C}$-PIB PET and 18F-FDG PET for detection of Alzheimer's disease, $\mathrm{J} \mathrm{Nucl}$ Med 48(4) (2007), 547-552.

[78] F. Nobili, D. Salmaso, S. Morbelli et al., Principal component analysis of FDG PET in amnestic MCI, Eur J Nucl Med Mol Imaging 35(12) (2008), 2191-2202.

[79] A. Nordberg, PET imaging of amyloid in Alzheimer's disease, Lancet Neurol 3(9) (2004), 519-527.

[80] R. Perneczky, A. Drzezga, J. Diehl-Schmid et al., Schooling mediates brain reserve in Alzheimer's disease: findings of fluoro-deoxy-glucose-positron emission tomography, $\mathrm{J} \mathrm{Neu}$ rol Neurosurg Psychiatry 77(9) (2006), 1060-1063.

[81] R.C. Petersen, Mild cognitive impairment as a diagnostic entity, J Intern Med 256(3) (2004), 183-194.

[82] R.C. Petersen, R. Doody, A. Kurz et al., Current concepts in mild cognitive impairment, Arch Neurol 58(12) (2001), 1985-1992.
[83] R.C. Petersen, G.E. Smith, S.C. Waring et al., Mild cognitive impairment: clinical characterization and outcome, Arch Neurol 56(3) (1999), 303-308.

[84] R.C. Petersen, J.C. Stevens, M. Ganguli et al., Practice parameter: early detection of dementia: mild cognitive impairment (an evidence -based review). Report of the Quality Standards Subcommittee of the American Academy of Neurology, Neurology 56(9) (2001), 1133-1142.

[85] J.R. Petrella, R.E. Coleman and P.M. Doraiswamy, Neuroimaging and early diagnosis of Alzheimer disease: a look to the future, Radiology 226(2) (2003), 315-336.

[86] M.E. Phelps, H.R. Schelbert and J.C. Mazziotta, Positron computed tomography for studies of myocardial and cerebral function, Ann Intern Med 98(3) (1983), 339-359.

[87] B.L. Plassman, K.M. Langa, G.G. Fisher et al., Prevalence of dementia in the United States: the aging, demographics, and memory study, Neuroepidemiology 29(1-2) (2007), 125-132.

[88] J.L. Price, A.I. Ko, M.J. Wade et al., Neuron number in the entorhinal cortex and CA1 in preclinical Alzheimer disease, Arch Neurol 58(9) (2001), 1395-1402.

[89] E.M. Reiman, Linking brain imaging and genomics in the study of Alzheimer's disease and aging, Ann N Y Acad Sci 1097 (2007), 94-113.

[90] E.M. Reiman, R.J. Caselli, K. Chen et al., Declining brain activity in cognitively normal apolipoprotein E epsilon 4 heterozygotes: A foundation for using positron emission tomography to efficiently test treatments to prevent Alzheimer's disease, Proc Natl Acad Sci U S A 98(6) (2001), 3334-3339.

[91] E.M. Reiman, R.J. Caselli, L.S. Yun et al., Preclinical evidence of Alzheimer's disease in persons homozygous for the epsilon 4 allele for apolipoprotein E, $N$ Engl J Med 334(12) (1996), 752-758.

[92] E.M. Reiman, K. Chen, G.E. Alexander et al., Functional brain abnormalities in young adults at genetic risk for late onset Alzheimer's dementia, Proc Natl Acad Sci U S A 101(1) (2004), 284-289.

[93] E.M. Reiman, K. Chen, G.E. Alexander et al., Correlations between apolipoprotein E epsilon4 gene dose and brainimaging measurements of regional hypometabolism, Proc Natl Acad Sci U S A 102(23) (2005), 8299-8302.

[94] M.L. Ries, C.M. Carlsson, H.A. Rowley et al., Magnetic resonance imaging characterization of brain structure and function in mild cognitive impairment: a review, J Am Geriatr Soc 56(5) (2008), 920-934.

[95] A.B. Rocher, F. Chapon, X. Blaizot et al., Resting-state brain glucose utilization as measured by PET is directly related to regional synaptophysin levels: a study in baboons, Neuroimage 20(3) (2003), 1894-1898.

[96] C.C. Rowe, S. Ng, U. Ackermann et al., Imaging betaamyloid burden in aging and dementia, Neurology $\mathbf{6 8}(20)$ (2007), 1718-1725.

[97] E. Salmon, B. Sadzot, P. Maquet et al., Differential diagnosis of Alzheimer's disease with PET, J Nucl Med 35(3) (1994), 391-398.

[98] M. Samuraki, I. Matsunari, W.P. Chen et al., Partial volume effect-corrected FDG PET and grey matter volume loss in patients with mild Alzheimer's disease, Eur J Nucl Med Mol Imaging 34(10) (2007), 1658-1669.

[99] D.J. Selkoe, Soluble oligomers of the amyloid beta-protein impair synaptic plasticity and behavior, Behav Brain Res 192(1) (2008), 106-113.

[100] D.H. Silverman, J.L. Cummings, G.W. Small et al., Added clinical benefit of incorporating 2-deoxy-2-[18F]fluoro-Dglucose with positron emission tomography into the clini- 
cal evaluation of patients with cognitive impairment, Mol Imaging Biol 4(4) (2002), 283-293.

[101] D.H. Silverman, S.S. Gambhir, H.W. Huang et al., Evaluating early dementia with and without assessment of regional cerebral metabolism by PET: a comparison of predicted costs and benefits, $J$ Nucl Med 43(2) (2002), 253-266.

[102] D.H. Silverman, G.W. Small, C.Y. Chang et al., Positron emission tomography in evaluation of dementia: Regional brain metabolism and long-term outcome, Jama 286(17) (2001), 2120-2127.

[103] J.D. Sluimer, W.M. van der Flier, G.B. Karas et al., Wholebrain atrophy rate and cognitive decline: longitudinal MR study of memory clinic patients, Radiology 248(2) (2008), 590-598.

[104] G.W. Small, Positron emission tomography scanning for the early diagnosis of dementia, West J Med 171(5-6) (1999), 293-294.

[105] G.W. Small, L.M. Ercoli, D.H. Silverman et al., Cerebral metabolic and cognitive decline in persons at genetic risk for Alzheimer's disease, Proc Natl Acad Sci U S A 97(11) (2000), 6037-6042.

[106] G. Spulber, E. Niskanen, S. Macdonald et al., Whole brain atrophy rate predicts progression from MCI to Alzheimer's di- sease, Neurobiol Aging (2008).

[107] Y. Stern, What is cognitive reserve? Theory and research application of the reserve concept, J Int Neuropsychol Soc 8(3) (2002), 448-460.

[108] J. Valla, J.D. Berndt and F. Gonzalez-Lima, Energy hypometabolism in posterior cingulate cortex of Alzheimer's patients: superficial laminar cytochrome oxidase associated with disease duration, J Neurosci 21(13) (2001), 4923-4930.

[109] L.A. van de Pol, E.S. Korf, W.M. van der Flier et al., Magnetic resonance imaging predictors of cognition in mild cognitive impairment, Arch Neurol 64(7) (2007), 1023-1028.

[110] W.M. van der Flier, F. Barkhof and P. Scheltens. Shifting paradigms in dementia: toward stratification of diagnosis and treatment using MRI, Annals of the New York Academy of
Sciences 1097 (2007), 215-224.

[111] V.L. Villemagne, C.C. Rowe, S. Macfarlane et al., Imaginem oblivionis: the prospects of neuroimaging for early detection of Alzheimer's disease, J Clin Neurosci 12(3) (2005), 221230.

[112] P.J. Visser, F.R. Verhey, P.A. Hofman et al., Medial temporal lobe atrophy predicts Alzheimer's disease in patients with minor cognitive impairment, J Neurol Neurosurg Psychiatry 72(4) (2002), 491-417.

[113] L.O. Wahlund, O. Almkvist, K. Blennow et al., Evidencebased evaluation of magnetic resonance imaging as a diagnostic tool in dementia workup, Top Magn Reson Imaging 16(6) (2005), 427-437.

[114] K.B. Walhovd, A.M. Fjell, I. Amlien et al., Multimodal imaging in mild cognitive impairment: Metabolism, morphometry and diffusion of the temporal-parietal memory network, Neuroimage (2008).

[115] I. Yakushev, A. Hammers, A. Fellgiebel et al., SPM-based count normalization provides excellent discrimination of mild Alzheimer's disease and amnestic mild cognitive impairment from healthy aging, Neuroimage 44(1) (2009), 4350.

[116] Y. Yuan, Z.X. Gu and W.S. Wei, Fluorodeoxyglucosepositron-emission tomography, single-photon emission tomography, and structural MR imaging for prediction of rapid conversion to Alzheimer disease in patients with mild cognitive impairment: a meta-analysis, AJNR Am J Neuroradiol 30(2) (2009), 404-410.

[117] J. Zaccai, C. McCracken and C. Brayne, A systematic review of prevalence and incidence studies of dementia with Lewy bodies, Age Ageing 34(6) (2005), 561-566.

[118] E. Zamrini, S. De Santi and M. Tolar, Imaging is superior to cognitive testing for early diagnosis of Alzheimer's disease, Neurobiol Aging 25(5) (2004), 685-691.

[119] K. Ziegler-Graham, R. Brookmeyer, E. Johnson et al., Worldwide variation in the doubling time of Alzheimer's disease incidence rates, Alzheimers Dement 4(5) (2008), 316-323. 


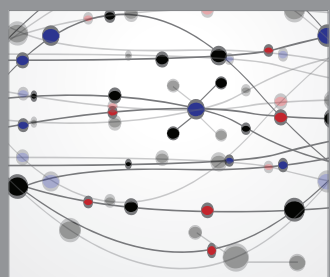

The Scientific World Journal
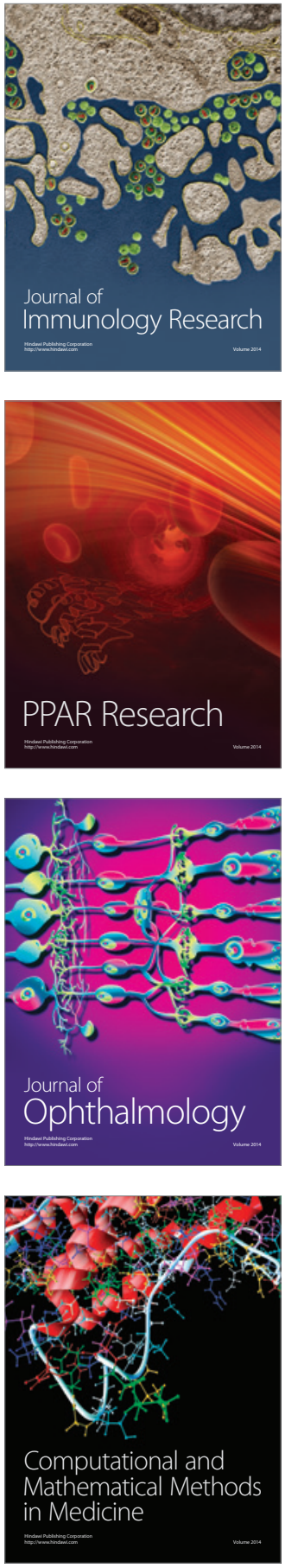

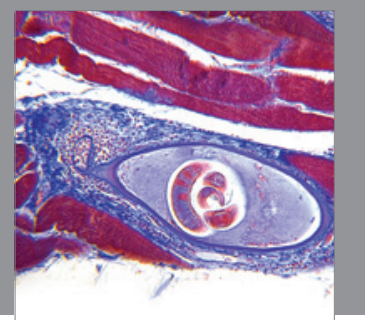

Gastroenterology

Research and Practice
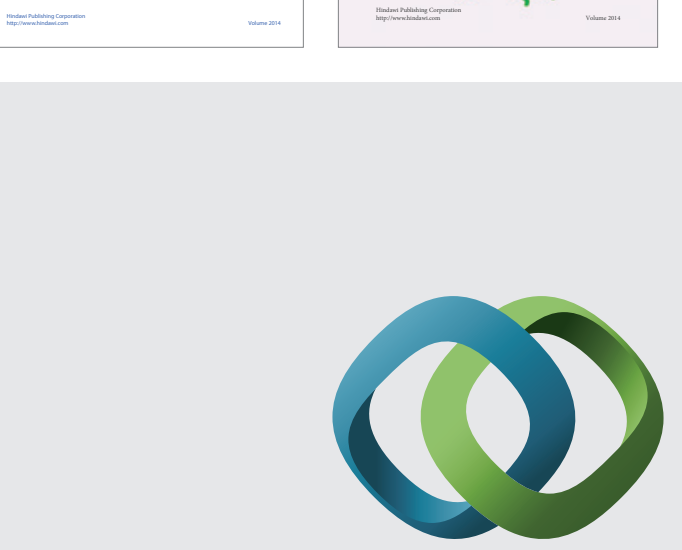

\section{Hindawi}

Submit your manuscripts at

http://www.hindawi.com
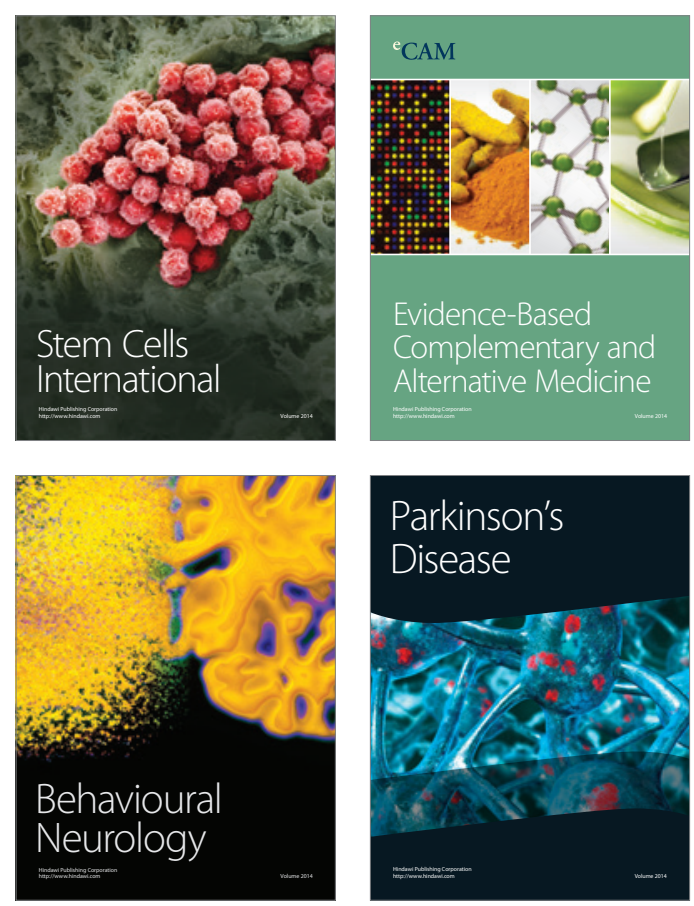

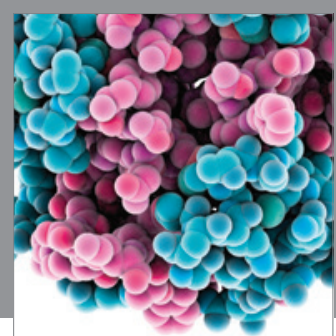

Journal of
Diabetes Research

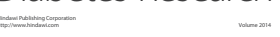

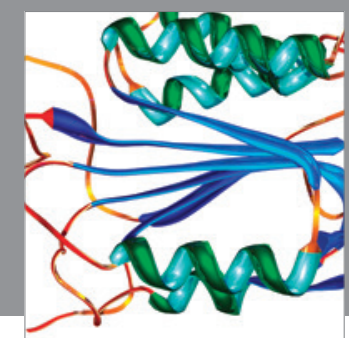

Disease Markers
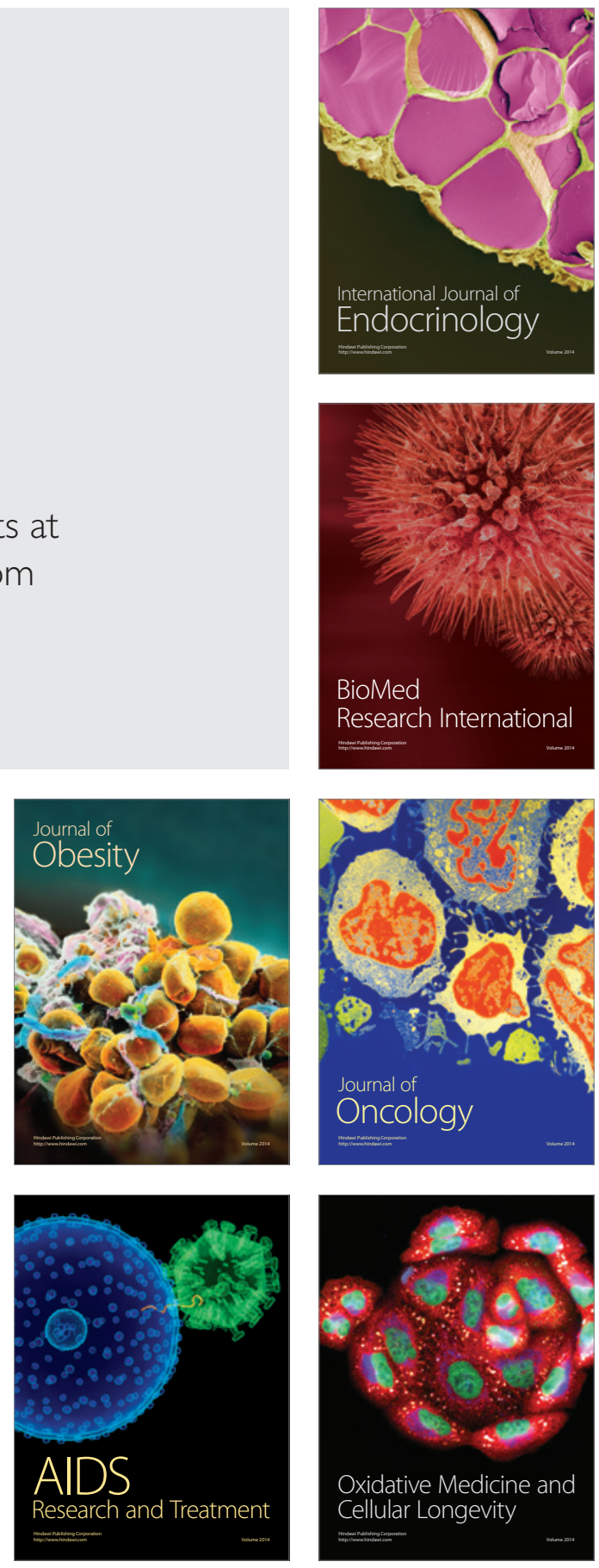